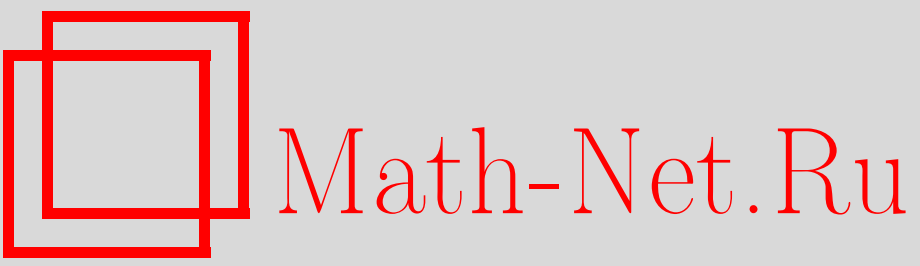

В. Н. Соловьев, Минимаксное и минимаксно-байесовское оценивание параметров линейной регрессии, УМН, 1996, том 51, выпуск 3, 211-212

DOI: https://doi.org/10.4213/rm989

Использование Общероссийского математического портала Math-Net.Ru подразумевает, что вы прочитали и согласны с пользовательским соглашением

http://www.mathnet.ru/rus/agreement

Параметры загрузки:

IP : 54.198 .55 .26

26 апреля 2023 г., 08:14:27 


\title{
МИНИМАКСНОЕ И МИНИМАКСНО-БАЙЕСОВСКОЕ ОЦЕНИВАНИЕ ПАРАМЕТРОВ ЛИНЕЙНОЙ РЕГРЕССИИ
}

\author{
В. Н. СОЛОвьЕв
}

1. Минимаксные оценки точечных параметров. Пусть в линейной регрессионной модели

$$
\mathscr{Y}=\mathscr{A} \theta+\xi, \quad \mathscr{Y}=\left(\begin{array}{c}
\gamma \\
\theta_{0}
\end{array}\right), \quad \mathscr{A}=\left(\begin{array}{c}
A \\
I_{m}
\end{array}\right), \quad \xi=\left(\begin{array}{c}
\rho \\
-\varepsilon
\end{array}\right)
$$

наряду с косвенными наблюдениями $\gamma=A \theta+\rho \in \mathbb{R}^{n}$ имеются прямые наблюдения $\theta=\theta_{0}+\varepsilon$ параметров $\theta \in \mathbb{R}^{m}$, а их ошибки удовлетворяют априорным ограничениям

$$
\mathrm{E} \xi=0, \quad \operatorname{cov}(\xi, \xi)=X \in \mathscr{X},
$$

в которых $\mathscr{X}$ - выпуклый компакт положительно определенных матриц порядка $n+m$. Рассмотрим линейные оценки $\widetilde{l}_{j}=\left(x_{j}, \mathscr{Y}\right), x_{j} \in \mathbb{R}^{n+m}$, параметров $l_{j}=\left(c_{j}, \theta\right), j=1, \ldots, r$. Обозначим через $x$ блочный вектор $x=\left\{x_{1}, \ldots, x_{r}\right\} \in \mathbb{R}^{(n+m) r}$. Гарантированное значение $2 D(x):=\sup _{\theta, \xi} \mathrm{E}|\widehat{l}-l|^{2}$ среднеквадратичной ошибки оценивания вектора $l=C \theta \in \mathbb{R}^{r}$ конечно, если $\mathscr{A}^{T} x_{j}=c_{j}, j=1, \ldots, r$. В этом случае

$$
2 D(x)=\max _{X \in \mathscr{X}} \sum_{j=1}^{r}\left(X x_{j}, x_{j}\right) .
$$

Чтобы найти минимаксные оценки $\widetilde{l}_{j}^{*}=\left(x_{j}^{*}, \mathscr{Y}\right)$ параметров $l_{j}, j=1, \ldots, r$, нужно решить задачу

$$
\widetilde{D}_{\min } / 2=\min _{x}\left\{D(x) \mid \mathscr{A}^{T} x_{j}=c_{j}, j=1, \ldots, r\right\} .
$$

Фундаментальную роль играет двойственная задача [1], [2]

$$
\widetilde{D}_{\min } / 2=-\min _{\theta_{j} \in \mathbb{R}^{m}}\left\{D^{*}\left(\mathscr{A} \theta_{1}, \ldots, \mathscr{A} \theta_{r}\right)-\sum_{j=1}^{r}\left(c_{j}, \theta_{j}\right)\right\} .
$$

Лемма. Сопряженная функиия $D^{*}(y)$ дифференцируема и может быть записана в форме

$$
D^{*}(y)=\min _{X \in \mathscr{X}} \sum_{j=1}^{r}\left(X^{-1} y_{j}, y_{j}\right) / 2 .
$$

Для фиксированного вектора $y=\left\{y_{1}, \ldots, y_{r}\right\} \in \mathbb{R}^{(n+m) r}$, матрица $X_{y} \in \mathscr{X}$ тогда и только тогда дает решение вспомогательной задачи (6), когда она дает решение задачи (3) для вектора $x=x(y)$ с компонентами $x_{j}(y)=X_{y}^{-1} y_{j}, j=1, \ldots, r . B$ этом cлучае $D^{*}(y)=D(x(y)), \nabla D^{*}(y)=x(y)$.

2. Теорема эквивалентности. Обозначим через $\mathscr{P}=\mathscr{P}_{0}$ класс всех распределений случайного вектора $Y=\{\gamma, \theta\}$, удовлетворяющих априорным ограничениям $\gamma=A \theta+\rho$, $\mathrm{E} \theta=\theta_{0}, \mathrm{E} \rho=0$ и $\operatorname{cov}(\xi, \xi)=X \in \mathscr{X}$ с вектором $\xi=\left\{\rho, \theta_{0}-\theta\right\}$. Пусть

$$
D_{\min }=\inf _{\widehat{l}(\cdot)} \sup _{P \in \mathscr{P}} \mathrm{E}_{P}|\widehat{l}-l|^{2}
$$

- это минимаксно-байесовская точность оценивания вектора $l=C \theta$ для класса $\mathscr{P}=\mathscr{P}_{0}$ и класса всех измеримых функций $\widehat{l}(\gamma): \mathbb{R}^{n} \rightarrow \mathbb{R}^{r}$, а $\widetilde{D}_{\min }-$ это минимаксная точность оценивания (4) точечных параметров $l$ в регрессионной модели $(1)-(2)$. Обозначим через $\widehat{l}_{*}$ и $\widetilde{l}_{*}$ решения задач $(7)$ и $(4)$, соответственно. 
Теорема 1. Если $\mathscr{X}$ - выпукльй компакт положстельно определенньх матриц, то

$$
D_{\min }=\widetilde{D}_{\min }=\max _{X \in \mathscr{X}} \sum_{j=1}^{r}\left(\left(\mathscr{A}^{T} X^{-1} \mathscr{A}\right)^{-1} c_{j}, c_{j}\right) .
$$

Пусть $X_{*} \in \mathscr{X}$ - решение задачи (8). Тогда МНК-оценка

$$
\widehat{l}_{*}=\widetilde{l}_{*}=C\left(\mathscr{A}^{T} X_{*}^{-1} \mathscr{A}\right)^{-1} \mathscr{A}^{T} X_{*}^{-1} \mathscr{Y}
$$

дает решение задач (7) и (4), а векторы

$$
\theta_{j}^{*}=\left(\mathscr{A}^{T} X_{*}^{-1} \mathscr{A}\right)^{-1} c_{j}, \quad j=1, \ldots, r
$$

дают решение двойственной задачи (5). И, наоборот, если $\theta^{*}$ - решение двойственной задачи, а $X_{*} \in \mathscr{X}$ - решение вспомогательной задачи (6), вычисленное для векторов $y_{j}=\mathscr{A} \theta_{j}^{*}, j=1, \ldots, r$, то $X_{*}$ - наименее благоприятная матрица ковариаций для МНК-оченок вектора $l$ (т.е. решение задачи (8)), и справедливы равенства (9), (10). Наименее благоприятно нормальное распределение $\xi \sim \mathcal{N}\left(0, X_{*}\right)$.

Tеорема 2. Если $\mathscr{P}=\mathscr{P}_{2}-$ класс всех распределений вектора ошибок $\xi$ со вторыми моментами $\mathrm{E} \xi \xi^{T} \in \mathscr{B}=\left\{B=X+e e^{T} \mid X \in \mathscr{X}, e \in \mathscr{E}\right\}$ где $\mathscr{E}$ - выпуклый чентрально-симметричный компакт в $\mathbb{R}^{m}$, то для задач (7) и (4) оченивания скалярного параметра $l=(c, \theta)$ справедливы равенства (8)-(10), если заменить матриць ковариачий $X \in \mathscr{X}$ на матрицы вторых моментов $B \in \mathscr{B}$. Наименее благоприятно нормальное распределение $\xi \sim \mathcal{N}\left(0, B_{*}\right)$.

3. Минимаксно-байесовские оценки параметров в случае произвольной корреляции сигнала и шума. Пусть теперь в модели наблюдений $\mathrm{E} \theta=\theta_{0}, \mathrm{E} \rho=0, \operatorname{cov}(\theta, \theta)=$ $T \in \mathscr{T}, \operatorname{cov}(\rho, \rho)=R \in \mathscr{R}$ нам ничего не известно о взаимной ковариации $\operatorname{cov}(\rho, \theta)$, кроме неотрицательной определенности матрицы ковариаций вектора $\{\theta, \rho\} \in \mathbb{R}^{n+m}$. Здесь мы будем считать, что ранги матриц $A$ и $C$ равны $m(r=m)$.

Tеорема 3. Справедливо равенство

$$
D_{\min }=\max _{D \geqslant 0, T \in \mathscr{T}, R \in \mathscr{R}}\left\{\operatorname{Sp} C D C^{T} \mid D \leqslant T, D \leqslant\left(A^{T} R^{-1} A\right)^{-1}\right\} .
$$

Пусть $D_{*}, T_{*}, R_{*}$ - решение задачи (11), $\widehat{l}_{1}=C\left(A^{T} R_{*}^{-1} A\right)^{-1} A^{T} R_{*}^{-1} \gamma-$ МнК-оченка вектора $l=C \theta, \widehat{l}_{0}=C \theta_{0}$ - априорная оценка и $D_{1}:=C\left(A^{T} R_{*}^{-1} A\right)^{-1} C^{T}, D_{0}:=C T_{*} C^{T}$ - ковариационные матрицы оценок $\widehat{l}_{1}$ и $\widehat{l}_{0}$. Тогда минимаксно-байесовской будет линейная оценка $\widehat{l}_{*}=P_{*} \widehat{l}_{1}+\left(I_{m}-P_{*}\right) \widehat{l}_{0}$, в которой $P_{*}-$ проектор $\left(P_{*}^{2}=P_{*}\right)$, вид которого указан ниже. При әтом

$$
C D_{*} C^{T}=P_{*} D_{1}+\left(I_{m}-P_{*}\right) D_{0}
$$

$u$ наименее благоприятно нормальное распределение вектора $\{\theta, \rho\}$ в случае $\operatorname{cov}(\theta, \theta)=T_{*}, \operatorname{cov}(\rho, \rho)=R_{*} u \operatorname{cov}(\rho, \theta)=-A D_{*}$.

Теорема 4. Проектор $P_{*}$ имеет вид $P_{*}=Q^{-1} P Q$, где матрица $Q$ удовлетворяет условиям $Q \geqslant I_{m}, Q C D_{*} C^{T}=C D_{*} C^{T}=P_{*} D_{1}+\left(I_{m}-P_{*}\right) D_{0} \geqslant 0$, a $P-$ ортогональный проектор на положительное собственное пространство матрицы $Q\left(D_{0}-D_{1}\right) Q^{T}$.

При $Q=I_{m}$ получаем ортогональный проектор $P_{*}=P$, если матрица $(12)$ неотрицательно определена. Интересно, что в этом случае апостериорная точность $\mathrm{E}_{P}|\widehat{l}-l|^{2}$ будет одной и той же для всех распределений $P(y)$ с фиксированными $\operatorname{cov}(\theta, \theta), \operatorname{cov}(\rho, \rho)$ и произвольной $\operatorname{cov}(\theta, \rho)$.

\section{СПИСОК ЛИТЕРАТУРЫ}

[1] Soloviov V. // Stochastics Stochastics Rep. 1993. V. 42. P. 209-223. [2] Soloviov V. // Anal. Math. 1993. V. 19. № 4. P. 297-315. 